\title{
SATURN
}

\section{Software Deobfuscation Framework Based on LLVM}

\author{
Peter Garba* \\ Thales, DIS - Cybersecurity \\ Munich, Germany \\ peter.garba@thalesgroup.com
}

\author{
Matteo Favaro \\ Zimperium, Mobile Security \\ Noale, Italy \\ matteo.favaro@reversing.software
}

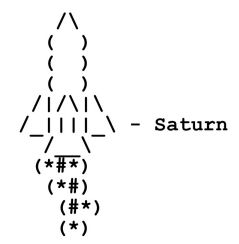

\begin{abstract}
The strength of obfuscated software has increased over the recent years. Compiler based obfuscation has become the de facto standard in the industry and recent papers also show that injection of obfuscation techniques is done at the compiler level. In this paper we discuss a generic approach for deobfuscation and recompilation of obfuscated code based on the compiler framework LLVM. We show how binary code can be lifted back into the compiler intermediate language $L L V M-I R$ and explain how we recover the control flow graph of an obfuscated binary function with an iterative control flow graph construction algorithm [3] based on compiler optimizations and satisfiability modulo theories (SMT) solving. Our approach does not make any assumptions about the obfuscated code, but instead uses strong compiler optimizations available in LLVM and Souper Optimizer to simplify away the obfuscation. Our experimental results show that this approach can be effective to weaken or even remove the applied obfuscation techniques like constant unfolding, certain arithmetic-based opaque expressions, dead code insertions, bogus control flow or integer encoding found in public and commercial obfuscators. The recovered $L L V M-I R$ can be further processed by custom deobfuscation passes that are now applied at the same level as the injected obfuscation techniques or recompiled with one of the available $L L V M$ backends. The presented work is implemented in a deobfuscation tool called SATURN (Figure 1).
\end{abstract}

\section{KEYWORDS}

reverse engineering, llvm, code lifting, obfuscation, deobfuscation, static software analysis, binary recompilation, binary rewriting

\section{INTRODUCTION}

In recent years we observed the increase in popularity and rise of intermediate language and source-based obfuscators, specifically due to the growth and diverse landscape of target architectures, especially on the mobile market [11]. While classical binary-based obfuscations were previously attacked by applying pattern-based rules or simple static analysis, higher-level obfuscations applied on intermediate language or source code cannot be effectively compromised. Modern protection tools are mostly based on state-of-theart compiler frameworks like $L L V M$ that allow much more complex

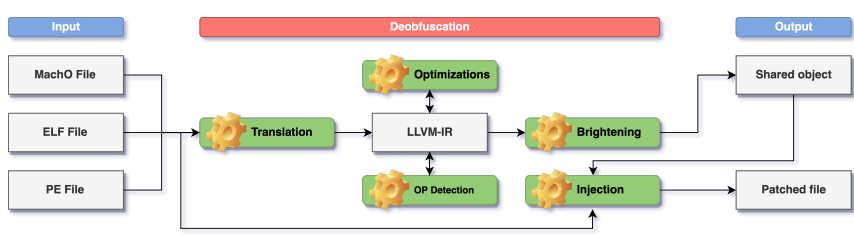

Figure 1: Workflow of the SATURN deobfuscation framework

obfuscation logic [11] [23].

In this paper we present an automatic deobfuscation approach based on $L L V M$ 's strong code optimizations. The paper focuses on several aspects that need to be addressed during deobfuscation: translation of binary code to LLVM-IR, control flow graph recovery, detection of opaque predicates, deobfuscation, brightening of the recovered function and recompilation.

Translating binary code into $L L V M-I R$ is not a straightforward task. A binary opcode does not only execute the operation itself, but might also address several other operations like the calculation of the condition codes/flags that influence later branch instructions. The information that could be used to translate the binary code into an intermediate language like the $L L V M-I R$ is normally lost during compilation and, especially in obfuscated binary code, this task can be even harder. One approach to target the problem is to implement the exact semantic of each binary opcode and store the output into a structure that holds the current state of the registers. This is a generic approach that lifts the binary code into a virtualized context but does not make any assumptions about the binary code itself. The recovered $L L V M-I R$ is fully functional but the readability of the IR might be very low. In this paper we make use of Remill [21] [14] to address the problem of binary code translation.

Control flow obfuscation is a technique to hide the original control flow of a function. To deobfuscate the function the attacker has to recover the control flow graph from the obfuscated binary code. Modern obfuscation tools that operate on intermediate languages 
like $L L V M$-IR have the ability to heavily obfuscate the control flow graph. We introduce an algorithm that makes use of the State struct in Remill to recover the edges of each lifted basic block. The lifted basic blocks and edges represent the recovered control flow graph. The recovery of the control flow graph is done statically and automatically during the lifting of the obfuscated binary code. Compared to previous work ([13], [37], [12], [35], [26]) that was done on control flow graph recovery, our approach does not need any prior knowledge about the binary code and doesn't rely on traces of the function. Instead, the path exploration is done based on the partially deobfuscated basic blocks and their predecessors. Our algorithm is similar to the Iterative Control Flow Graph Construction in [3] but is superior in the way that it works independently of the order in which the branches are examined.

A technique to conceal the control flow graph of a function is the insertion of opaque predicates (OP) to thwart naïve control flow graph reconstruction algorithms. An opaque predicate is a conditional branch injected into the control flow graph whose condition exists to confuse or thwart reverse engineering, but whose evaluation is deterministic, and thus irrelevant to the greater logic of the program [7]. We present an effective approach to detect and remove opaque predicates. The shown approach is based on strong LLVM and Souper Optimizer optimizations. For opaque predicates that are resistant to the applied optimizations and/or to verify the optimization results, we use an approach based on SMT solving. The way to identify opaque predicates with $S M T$ solving is not new [19], but we believe that the way we combine several tools and algorithms are a rich contribution to this paper.

Brightening [COMP.] verb - Reshaping code to make it more readable and understandable for humans

Constant unfolding, arithmetic-based opaque expressions, dead code, bogus control flow and integer encoding are not only found in hardened code, but can also appear in non-obfuscated code. Normally, during compilation of the source code, the compiler detects this kind of patterns and optimizes them away to obtain the best possible result. The presented approach relies on the reshaping of the $L L V M-I R$, as the way the code gets lifted by Remill might hinder the optimizer to reach the best result. The needed steps to reshape the $L L V M-I R$ are generic and don't rely on any prior knowledge about the obfuscator.

Without brightening, the $L L V M-I R$ would be fully functional but in this paper we aim to reach a vanilla ${ }^{1}$ state representation of the lifted function. This includes reconstruction of the original function arguments and transformation of the Remill specific lifted function based on the State struct (Listing 1) into a clean LLVM function with its original signature.

Once the control flow graph is recovered and the function is deobfuscated, one of the goals of the presented approach is to recompile and execute the lifted function. Due to the choice of $L L V M-I R$ as destination language for the lifted binary code, we can easily compile the recovered code back into binary code by using one of the

\footnotetext{
${ }^{1}$ As close as possible to a non-obfuscated compiled source code
}

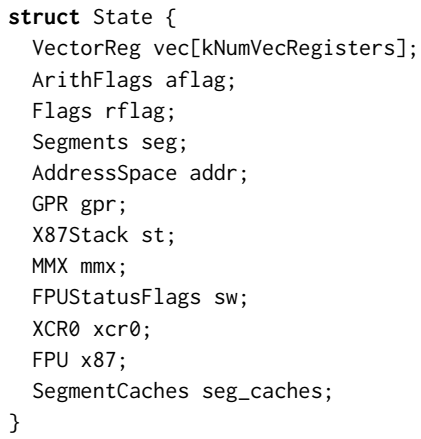

Listing 1: Remill State struct definition for x86_64

available $L L V M$ backends (X86, ARM, AArch64, RISC-V and others).

Our experiments show that we are able to apply our approach on current state-of-the-art obfuscations and also, to partially defeat the anti-symbolic deobfuscation tricks introduced in [22].

Our work is not only useful for deobfuscation. In fact this approach can also be used for further applications like fuzzing, as input for dynamic symbolic execution (DSE) engines like KLEE [4], as input for $L L V M$ based obfuscators like $O-L L V M$ [11], to achieve automatic payload creation for exploitation as shown in [34] or in general to recompile binary code with the best available $C P U$ optimizations $(-$ march=native) to improve the performance of applications or to introduce new compiler based security features. This applies especially to applications where the source code is not available.

\subsection{Goals and Challenges}

We want to propose a deobfuscation framework based on $L L V M$ and its strong optimizations for real world applications. Using $L L V M$ for reverse engineering might look like an overcomplication in the beginning, but it's similar to what is done during the compilation of source code. The $L L V M$ compiler framework has all the needed tools to easily create and modify the control flow graph, its basic blocks and instructions. The challenge is to lift the binary code into the LLVM-IR and get it into a shape that is equal to a nonobfuscated compiled source code. The techniques to reach this goal should be generic, non error-prone and lightweight. The framework should always generate working LLVM-IR that can be recompiled and executed. We aim at proposing a framework to lift the binary code back into a clean and understandable $L L V M-I R$ that is built on mature tools around the $L L V M$ ecosystem. Our vision is to get the attack surface back to the level it was implemented at - the compiler level.

\subsection{Contribution}

We summarize our contributions as follows.

- We propose an automatic deobfuscation tool that is generic enough to deal with several obfuscation techniques. 
- We propose a framework that can recompile and inject the $L L V M-I R$ code back into the given binary.

- We propose an effective and efficient method to identify opaque predicates at the LLVM-IR level, that are then solved and verified using compiler optimizations and SMT solvers.

- We propose a generic method to transform the binary code lifted by Remill into a cleaner LLVM-IR without the Remill State struct. This includes the recovery of the stack and the function arguments.

- We show that our work can be used to weaken or even remove anti-symbolic execution tricks like the ones introduced in the work of [22] and allows the usage of state-of-the-art source-level dynamic symbolic execution tools.

- We propose a framework that can generate a compact representation of the obfuscated constraints that are easier to solve or check for satisfiability.

\subsection{Discussion}

We explore several steps to recover the binary code from an obfuscated binary, based on the lifting of the code to the compiler intermediate language $L L V M-I R$. We propose several algorithms that were implemented in the tool SATURN that help to handle different aspects of binary code deobfuscation. To our knowledge the implementation of SATURN is state-of-the-art and lifts the attacking surface from the obfuscated binary code back to the compiler level. Our work has a high impact on the security of obfuscated binaries and allows the usage of efficient state-of-the-art source-/IR-level dynamic symbolic execution tools like KLEE to further analyze the recovered code. We provide an experimental setup that includes several corner cases that might hinder binary code lifting, but also apply our method to strong obfuscated real world binaries.

\section{BACKGROUND}

\subsection{LLVM}

" $L L V M$ began as a research project at the University of Illinois, with the goal of providing a modern, SSA-based compilation strategy [33] capable of supporting both static and dynamic compilation of arbitrary programming languages. Since then, LLVM has grown to be an umbrella project consisting of a number of sub-projects, many of which are being used in production by a wide variety of commercial and open source projects as well as being widely used in academic research" [16]. To understand our approach it's not crucial to understand how $L L V M$ and its internal language $L L V M-I R$ are designed, but the reader should keep in mind that the $L L V M-I R$ is based on the Static Single Assignment form (SSA) [8] which makes it easier to construct the final formula passed to the SMT solver [34].

\subsection{Remill}

"Remill is a static binary translator that translates machine code instructions into $L L V M$ bitcode. It translates $\mathrm{x} 86$ and amd64 machine code (including AVX and AVX512) into the LLVM-IR" [21]. In our work we make extensive use of Remill to lift the binary code into the LLVM-IR. Remill does not make any assumptions about the stack or the arguments of a lifted function since it only lifts single instructions.

\subsection{Souper optimizer}

Souper is particularly convenient because it's an $L L V M$-based project that, with the help of KLEE [4], is capable of converting a sequence of $L L V M-I R$ instructions into an SMT formula and use several SMT solvers to discover additional peephole optimizations. As a desired side-effect we can benefit from its results to determine the opaqueness of a conditional branch. Souper has the possibility to cache the SMT queries and results into an external Redis database [24] to improve the performance. This leaves us with a database full of opaque predicates and obfuscation patterns that could be analyzed in further studies.

\subsection{KLEE}

"KLEE is a symbolic execution tool capable of automatically generating tests that achieve high coverage on a diverse set of complex and environmentally-intensive programs and operates on the $L L V M$ $I R "$ " [4]. KLEE is not only a useful tool for testing software but it's also very effective in attacking several code obfuscation techniques. Current work proposed in [22] tries to hinder symbolic execution tools like KLEE to do their work effectively.

\section{MOTIVATION}

\subsection{Attacker model}

Goal. We consider a man-at-the-end (MATE) scenario where the attacker has full access to a protected binary under attack and no access to the source code or unprotected binary. The attack model and the methodology follow closely the survey by Schrittweiser et al. [28] and are similar to the ones considered in [22]. To be more concrete, we will focus on the following goals: 1. Recovery of the control flow graph. Retrieving the control flow graph of an obfuscated function is a crucial step to understand what the original function performs. 2. Detection of opaque predicates. Recovery of the control flow graph can only be successful if the injected opaque predicates can be detected and removed. 3. Deobfuscation of several obfuscation techniques. To make the code readable and understandable the injected obfuscation patterns have to be detected and removed. 4. Recovery of the stack and arguments. If the attacker can rebuild the stack and arguments, the code of the function will become tidy. 5. Execution of the recovered code. If the attacker is able to execute a semantically equivalent deobfuscated code, further analysis can be done with tools like a debugger if needed.

\subsection{Motivating example}

Let us illustrate some anti-symbolic path-oriented protections on a toy program like those introduced in the work of [22]. Listing 2 displays an unoptimized obfuscation of a simple toy example that is protected against symbolic execution attacks with the FOR and SPLIT tricks as introduced in [22] and extended with an opaque predicate to protect the final calculation. 


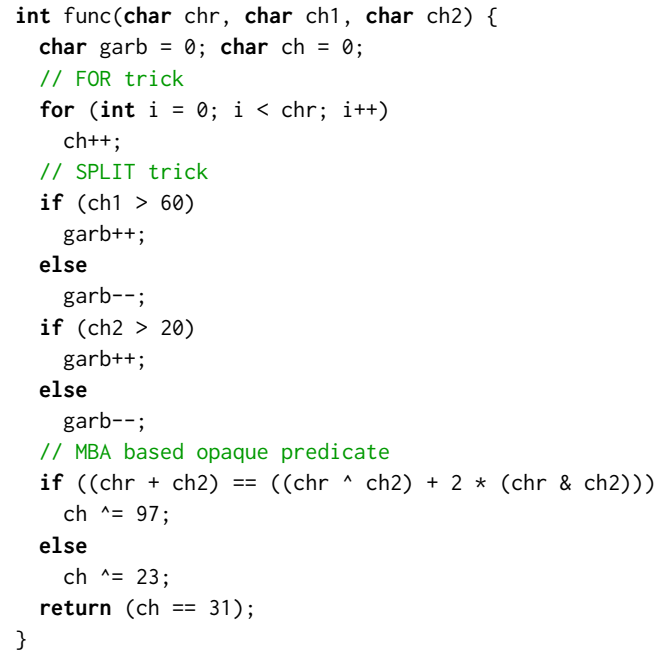

Listing 2: Anti-symbolic path-oriented protections FOR and SPLIT applied on a toy program based on [22]

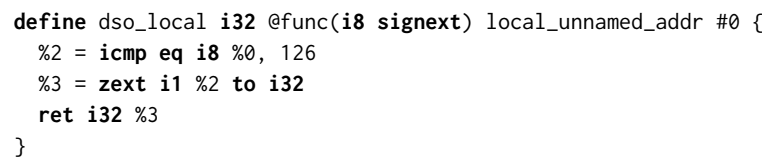

Listing 3: Unprotected toy program compiled to LLVM-IR

The anti-symbolic tricks that we considered are not resistant to compiler optimizations and can easily be removed by compiling the code with clang -03 optimization. The introduced opaque predicate is resistant to compiler optimizations and can only be recovered by SMT solving. In our tests we will compile the toy example with clang -OO to hinder the optimizer from optimizing away the introduced tricks. The output binary therefore contains several stack slots, that are required to be recovered during the brightening step. If we fail to recover the stack slots and arguments, the $L L V M$ optimizations will fail to work and the anti-symbolic tricks won't be removed. If we succeed, the retrieved $L L V M-I R$ should look similar to the output of clang -O3 -S -emit-llvm in Listing 3 applied on the toy program without any obfuscation.

\section{FUNCTION RECOVERY}

Two of the core features of SATURN are the exploration and control flow graph reconstruction phases. The $L L V M$ ecosystem relies on powerful and correct algorithms that we made use of during the development of SATURN's passes. In this section we explain how SATURN achieves full function recovery starting from the binary code.

\subsection{Code lifting to LLVM-IR}

SATURN heavily relies on Remill. That's why it's important to understand how Remill is lifting a native instruction to LLVM-IR. Remill makes use of the target architecture's CPU instruction semantics to lift an instruction. In Listing 1 we can see the State struct for the $x 86 \_64$ architecture.

To emulate an x86_64 instruction like add rax, rcx Remill will create a call to a helper function that implements the emulation for the instruction. This function takes the State variable as an argument (Listing 4) and calculates the result according to the semantic of the instruction. This also includes the Flags registers. Once all instructions of a basic block are lifted, the generated calls get inlined into the caller. The output $L L V M-I R$ is not very readable at this step, but it behaves functionally the same as the native counterpart.

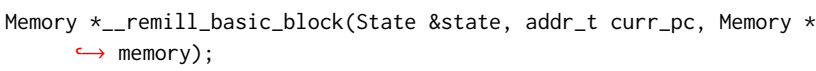

Listing 4: Remill basic block function signature $\mathrm{C} / \mathrm{C}++$

During the lifting, SATURN stores each recovered basic block into its own $L L V M-I R$ function. The basic block functions then get connected in a separate $L L V M-I R$ function, which is representing the recovered control flow graph (Figure 2).

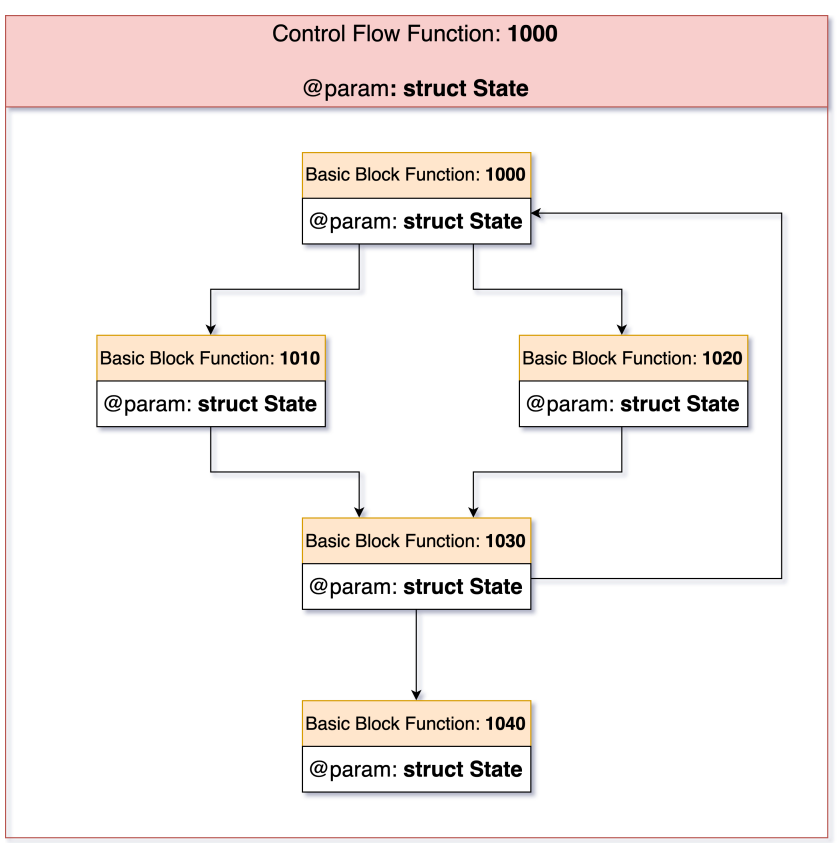

Figure 2: $L L V M$ function that contains the control flow graph of the recovered function at address 1000 . The basic blocks are lifted as $L L V M$ functions themselves and get called according to their usage. The result of the call decides the destination of the branch.

With this design decision we can directly optimize the lifted basic block function and achieve a performance improvement in further deobfuscation steps. Applying optimizations at this step also removes some simple obfuscation patterns. The control flow 
function is kept as simple as possible, which allows us to add/remove edges without the need to change the lifted code and avoids dealing with LLVM's PHI-nodes [33].

SATURN decides how to proceed with the path exploration during the lifting of basic blocks. For that SATURN is using the Remill instruction categories that are generated for each lifted instruction. Based on the instruction category SATURN will try to detect if the basic block is ending with an opaque predicate (Table 1) or not. If the basic block is a candidate for an opaque predicate, SATURN will first try to prove the outgoing edges by applying LLVM's optimization passes. If after optimization the count of the outgoing edges is greater than one, SATURN will try to solve the outgoing edges by making use of Souper and the Z3 SMT solver [9]. SATURN is always trying to use $L L V M$ 's optimizations first, since they are much cheaper, performance-wise, compared to the use of an SMT solver. Our tests with various obfuscation engines show that most of the generated opaque predicates are not resistant to LLVM's optimizations. The handling of opaque predicates is well described in Section 5.

Table 1: Path Exploration

\begin{tabular}{lcc}
\hline kCategory & Exploration & Opaqueness Proof \\
\hline NoOp & Continue & No \\
Normal & Continue & No \\
FunctionReturn & Stop & Yes \\
IndirectJump & Stop & Yes \\
DirectJump & Stop & No \\
ConditionalBranch & Stop & Yes \\
IndirectFunctionCall & Stop & Yes \\
DirectFunctionCall & Continue & No \\
\hline
\end{tabular}

SATURN continues with the lifting process as long as new edges are discovered. When $S A T U R N$ is discovering a new incoming edge for a basic block, it has to prove that the new edge does not change the opaqueness of an already (temporarily) proven basic block (Table 1). The following steps are applied:

(1) create a new function, called FSlice, based on the definition in Listing 4

(2) find all the basic blocks that dominate the lifted basic block, that we identify as BBLift

(3) if more than one predecessor is found, stop and continue at step 5

(4) repeat step 2 - 3 with the predecessor as input and store the result in a sorted list, called PSort, based on the dominance

(5) for each predecessor in PSort create a call in FSlice in reverse order

(6) connect the called predecessors with a branch instruction

(7) call BBLift at the end of FSlice and connect it to the called dominating predecessor with a branch

(8) inline all calls in FSlice and apply the $L L V M$ optimizations.
Now SATURN is using one of the solutions explained in Section 5.3 to determine the opaqueness of the basic block.

The basic block opaqueness might change to non-opaque and help us to detect false positives. This step is important, as it guides further code exploration. We also need this step because we can't know about all the incoming edges in the beginning and we gain the needed knowledge about the control flow graph only during the exploration phase. The opaqueness of the basic block will change according to Table 2.

Table 2: Basic Block Opaqueness

\begin{tabular}{ccc}
\hline Current Opaqueness & New Edge & New Opaqueness \\
\hline No OP & No OP & No OP \\
No OP & OP & No OP \\
OP & No OP & No OP \\
OP & OP & OP \\
\hline
\end{tabular}

\section{DEOBFUSCATION BY OPTIMIZATION}

The capability to easily build custom optimization passes is part of the core design of $L L V M$. In this section we are going to cover the custom optimization passes that we implemented to facilitate the propagation of constants and the identification of opaque predicates.

\subsection{Constants}

Storing constants in data sections is a common obfuscation technique to trick disassemblers like IDA Pro [10] into generating wrong results or simply stop the disassembling of the function. During deobfuscation, SATURN tries to detect accesses to such constants and replace the read instruction with a constant value in the $L L V M$ $I R$. Demoting the global variables helps the $L L V M$ optimization passes to apply constant folding and defeat such kind of obfuscation tricks. The user has to supply the ranges where to look for such constant data with the SATURN option constantPool. Our tests with several obfuscators show that it's not enough to use the constant binary data sections. In the obfuscators we looked at, we could find sections with read/write attributes that contained such constants.

\subsection{Stack pointer aliasing}

Remill does not know about the concept of a stack. Instead of trying to emulate the stack, it handles the stack operations by using read and write intrinsics (Listing 5) relying on the stack register as address. The stack register is part of the State struct and is defined as an unsigned integer value like uint64_t State.gpr.rsp.qword for the $x 86 \_64$ architecture. In SATURN the access to the stack will be represented as a load/store of an IntToPtr value. This makes it impossible for $L L V M$ to apply pointer aliasing, because $L L V M$ does not support pointer aliasing on integer values [17].

In SATURN this problem is handled by concretizing the stack register in the function representing the control flow graph. We then inline the basic block functions and optimize the code. During 
uint $<T>$ _t__remill_read_memory_<T>(Memory *, addr_t $)$;

Memory *__remill_write_memory_ $\langle T\rangle\left(\right.$ Memory *, addr_t, uint $\left.\langle T\rangle \_t\right)$;

Listing 5: Remill's memory read and write intrinsics definition

optimization the concrete stack register value will be propagated through the $L L V M-I R$ and will replace the IntToPtr operand with a concrete memory location. This concrete value helps us to identify the stack slot. We then create a global variable and a $L L V M-I R$ Alloca instruction for the stack slot at the beginning of the control flow graph function. After that we load the value from the global variable and store it into the Alloca value right after the Alloca instruction. We keep a map of known stack slots, their global variables and of the generated Alloca instructions. We optimize the code again and now, based on the allocas, LLVM is able to apply a proper pointer aliasing pass. These steps may reveal new concrete stack slots and we repeat this algorithm until no new stack slots are detected. Once it's finished we remove the unused global variables.

After the algorithm is done, some global variables are not optimized away. These global variables represent the return value, the function arguments passed on the stack and the values popped from the stack and stored in the execution context by the function. This is a side effect that we can use in the further two deobfuscation steps code brightening and recovering of the function arguments.

Pointer aliasing on the stack is an important feature for deobfuscation. It's crucial that this step gives accurate results, since it's needed for the following optimization steps.

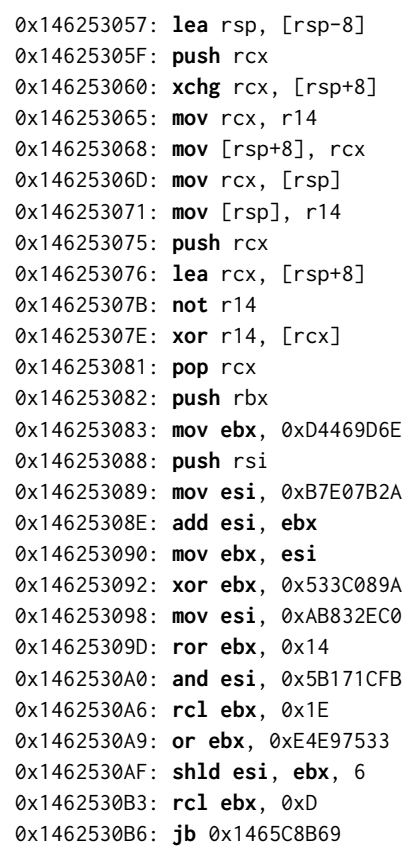

Listing 6: Obfuscated x86_64 opaque predicate

\subsection{Breaking Opaque Predicates with LLVM-IR Optimizations}

SATURN is approaching the opaque predicates problem in two steps. First it creates a slice of the instruction pointer and then applies LLVM optimizations on it. If the optimization is successful, the slice will fold into a single concrete value.

The available open source slicers ([38][5][29]) seem to be too outdated or unreliable to produce a valid slice for a given function. Conversely, our algorithm is based on modelling the slicing process in $\mathbf{C}$ and then relying on the $L L V M$ optimizations to produce the slice.

5.3.1 SATURN's slicing. The Remill's basic block definition in Listing 4 contains the information to control and inspect the value of a general purpose register before and after the execution of a Remill function. Based on the Remill basic block, the slicing is achieved with the following steps:

(1) initialize a Remill State struct with a symbolic state

(2) concretize the initial instruction pointer (RIP)

(3) call the opaque basic block, that has been previously optimized with the constant promotion and stack aliasing passes. This call is inlined during further optimization

(4) pass the initialized State struct to the basic block to be proven to be opaque

(5) get the resulting State struct after the basic block execution, specifically inspecting the final instruction pointer.

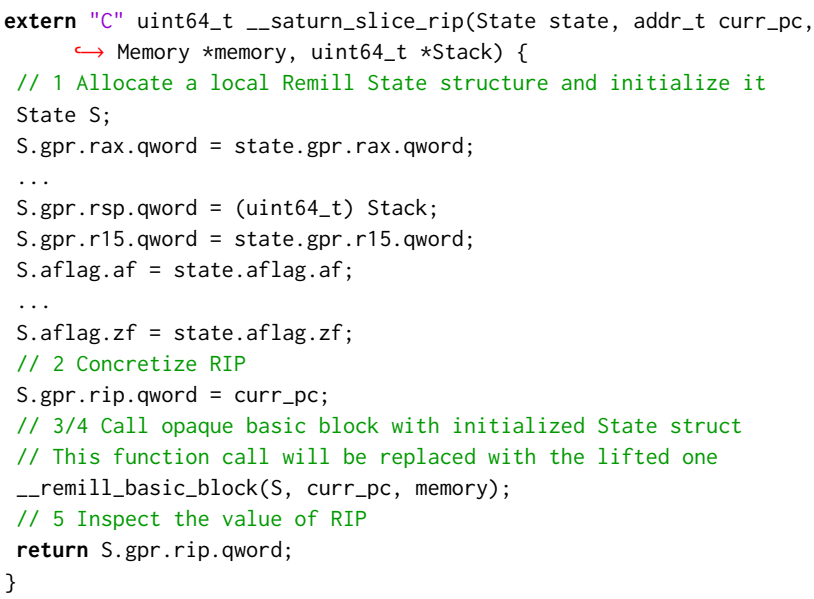

Listing 7: SATURN's slicing helper function

The initialization and further inspection steps are in Listing 7. The final step is taking the generated__saturn_slice_rip function and applying $L L V M$ optimizations on it. If the function implements an opaque predicate and $L L V M$ is able to optimize it away, the function will end with a concrete return value. This is the deterministic instruction pointer address where the basic block will continue. Listing 6 is showing an example of the obfuscated opaque predicate. In Listing 8 we can appreciate the result of the previously described 
process. The opaqueness has been broken and the unique destination address recovered.

SATURN has two options to control the amount of basic blocks to be used while slicing the value of the instruction pointer. This is needed as some obfuscators reuse values from previous basic blocks in subsequent opaque predicates. The SATURN options solverBBCount $7 c c$ and solverBBCountReturn let the user specify the amount of basic blocks with a single predecessor to connect to the current opaque block before optimizing it.

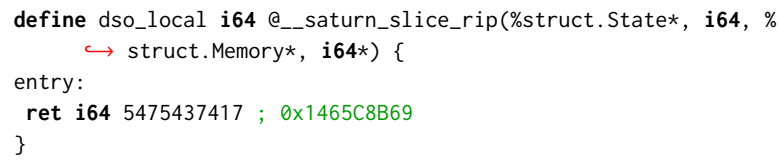

Listing 8: Sliced and optimized LLVM-IR with recovered Opaque Predicate offset 0x1465C8B69

In the next section we approach the problem of hard to optimize opaque predicates, most commonly based on Mixed-BooleanArithmetic (MBA [3]) expressions, as seen in our motivating example in Listing 2.

\subsection{Solving Opaque Predicates with Souper and $\mathrm{Z3}$}

The previous approach might fail because $L L V M$ 's optimizations are not successful in reducing the sliced instruction pointer to a constant. This means the conditional branch is either based on a stronger opaque predicate or might be a real conditional branch. To further analyze the branch we use the Souper Optimizer [27] and a $S M T$ solver. The steps taken to prove the opaqueness with the $Z 3$ theorem prover integrated within Souper are the following:

(1) extract the sliced instruction pointer value from the opaque basic block (Value \%17 in Listing 9)

(2) collect a set of candidate expressions to be solved by Souper

(3) select the Souper expression corresponding to the sliced instruction pointer value from the set

(4) build an SMT query that aims at finding one valid solution for the sliced instruction pointer expression

(5) if the query is not satisfiable, something went wrong in the proving process and the pass fails

(6) if the query is satisfiable, a valid solution for the expression has been discovered and a second SMT query is built to determine if the unique solution has been found, as shown in Listing 10

(7) if the last query is satisfiable, the conditional branch has been proven to be opaque and the real destination has been determined

(8) if the last query is not satisfiable, a real conditional branch or an opaque predicate which is not provable by the SMT solver has been found.

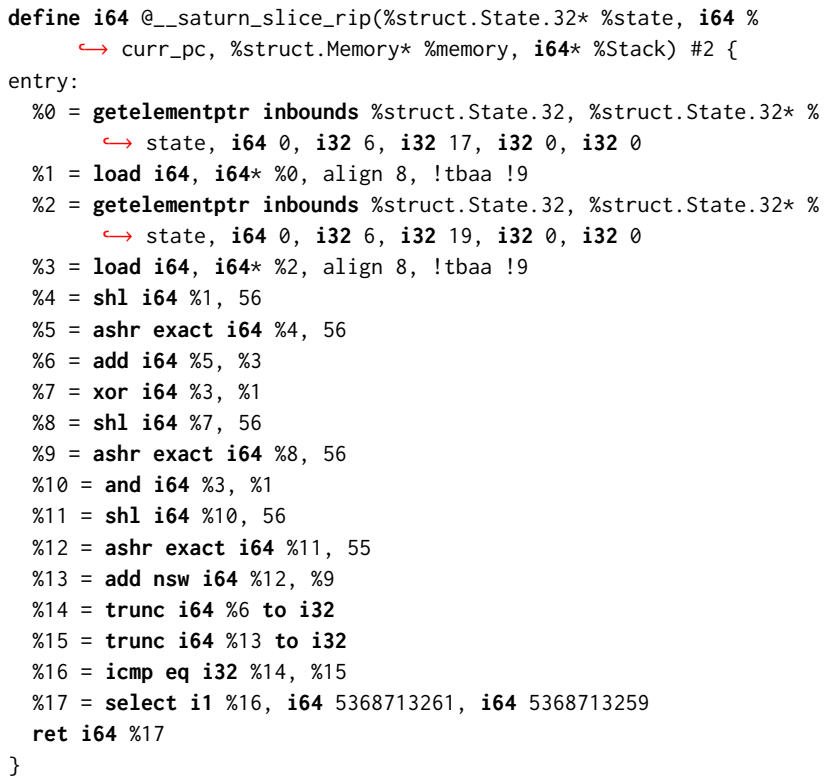

Listing 9: Sliced MBA of the toy program

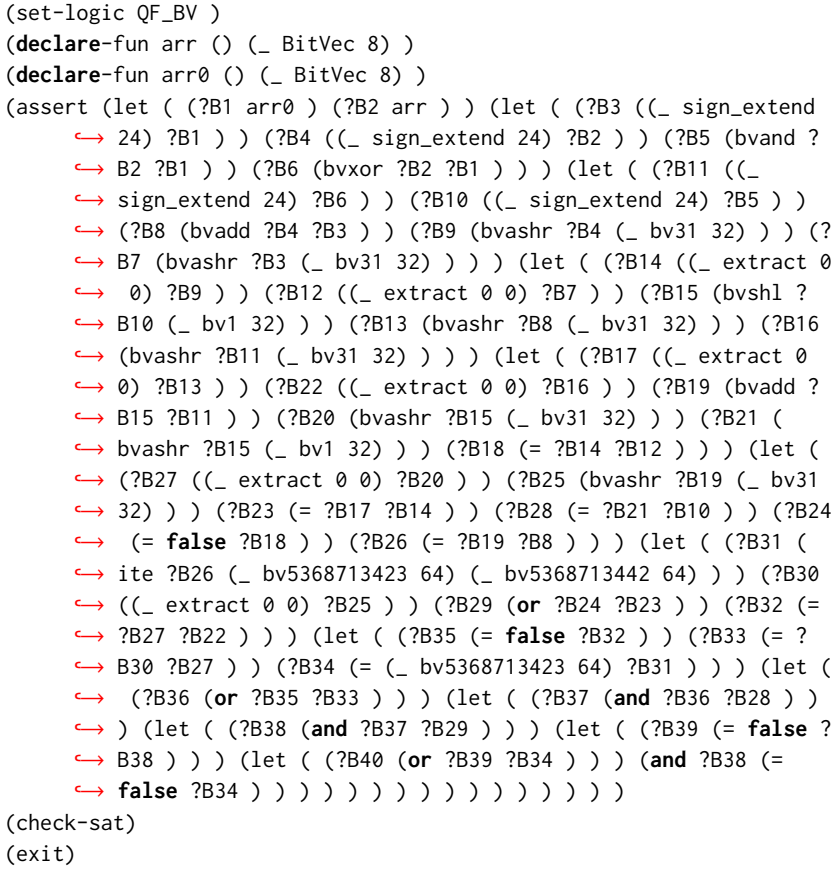

Listing 10: Z3 SMT query to solve the MBA opaque predicate from the toy program in Listing 2

\section{RECOMPILATION}

SATURN is not only lifting and deobfuscating the code, one of the goals is also to be able to recompile the LLVM-IR and make it executable. In this section we explain how SATURN is preparing the code for recompilation and how it achieves vanilla-like results. 


\subsection{Post Translation Optimization}

Once the obfuscated function is recovered, SATURN starts the post translation optimization phase, where the input is the control flow graph function shown in Figure 2. The steps are as follows:

(1) the stack register (RSP) and the instruction pointer register (RIP) contained in the State variable are concretized

(2) allocas are created for the flag calculation and stored into the State struct. This helps to optimize and remove unneeded flag calculations

(3) the basic block functions are inlined like seen in Figure 2

(4) the $L L V M$ optimizations are applied to the function

(5) the constant promotion algorithm (Section 5.1) and the stack alias analysis (Section 5.2) are applied to the function

(6) the steps 2-4 are repeated until no further changes are detected.

After the post translation is done, the output LLVM-IR is in a deobfuscated state but it's still difficult to understand the code because of the operations applied on the Remill State struct like shown in Listing 11. At this point the concretization of the registers can be removed and the $L L V M-I R$ can be compiled to binary code by making use of one of $L L V M$ 's backends. In the tests we use Clang to compile the output $L L V M-I R$ into a shared object. SATURN has two options to recompile the $L L V M-I R$ :

- the first option keeps the lifted function with the Remill signature as defined in Listing 4 . The created $\mathbf{C + +}$ helper functions do the context switch from the $x 86 \_64$ to the virtual context and take care of the State struct handling;

- the second option recovers the original function arguments and removes the State struct. This method has the benefit that the function can be called directly without a context switch. This approach is detailed in Section 6.2.

\subsection{Code Brightening}

The function lifted by Remill is operating on a virtual context (Listing 11), the State struct. This hinders the optimizer from detecting some optimization opportunities, as it has to store the results for each register back to the State struct. This happens for all the registers shown in Listing 11. At this point the output code is still too difficult to understand in further analysis steps like reverse engineering. In this section we address this problem and show how the original signature of the function can be reconstructed. This includes recovering the function arguments and removing the State struct, which leads to vanilla-like results.

6.2.1 Function Arguments. Based on the algorithm in Section 5.2, the arguments of a lifted function that are passed through the stack, are detected by inspecting the remaining global variables. During the execution of the algorithm in Section 5.2, SATURN keeps track of the global variables and their stack offsets. This information will be used to detect the number of arguments, with the knowledge about the application binary interface (ABI) and the calling convention [18] used by the function. If no stack arguments are passed to the function, we detect the number of arguments through the register accesses on the State struct. We only focus on the reconstruction of the general purpose registers in the following steps:

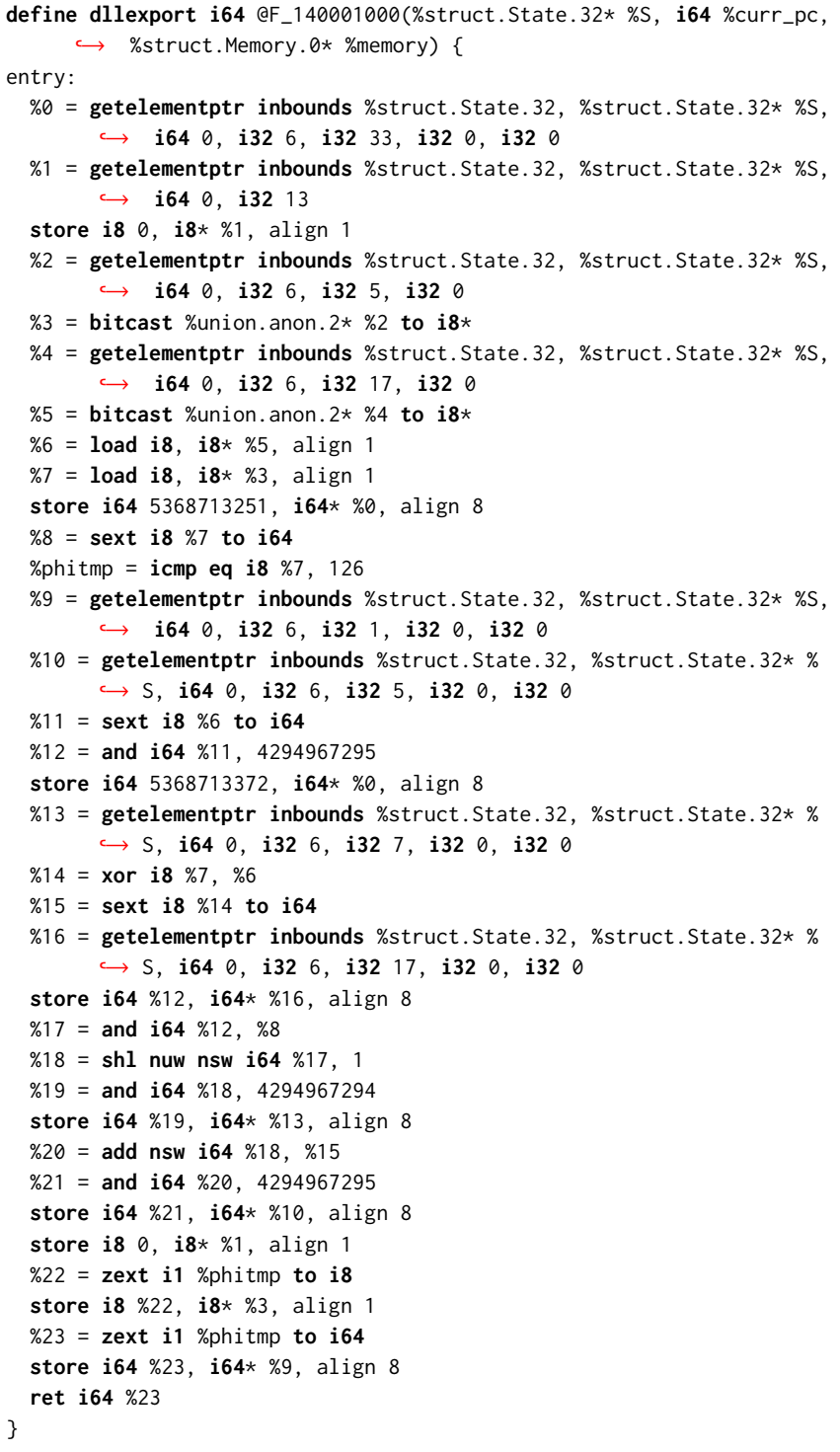

Listing 11: Recovered toy program LLVM-IR in the Remill State struct form

(1) based on the function's calling convention, start with the last register argument in the function's [18] argument list and search for the first getElementPtr (GEP) instruction that's accessing the register and is also dominating all the other GEP instructions that access that register

(2) if no GEP instruction is found, continue with the next register and decrease the number of arguments by one

(3) if a GEP instruction was found, forward slice the GEP value to get a tree of users that have a reference to the GEP instruction

(4) sort the users based on their position in the dominance tree DT of the function

(5) look for load and store instructions to detect how the GEP is used 
(6) if a dominating load or store can be found, assume that this register is an argument

(7) else decrease the number of arguments by one and continue at step 3 .

6.2.2 Function reconstruction. Based on the recovered number of arguments we start to rebuild the lifted function to be detached from the State struct. We use helper functions in $\mathbf{C} / \mathbf{C + +}$ that assist us to easily map the function arguments to their slots in the State struct as shown in Listing 12.

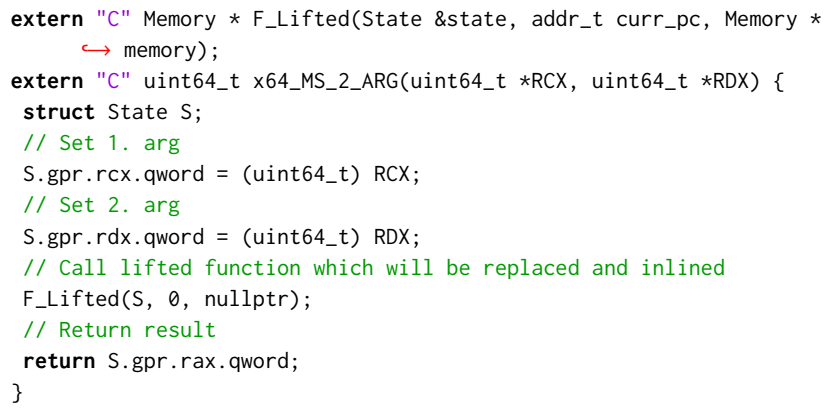

Listing 12: SATURN's helper $\mathrm{C} / \mathrm{C}++$ function to handle a Windows 64-bit $\mathrm{ABI}$ function with 2 arguments

We only need to prepare helpers for the register based arguments. On functions that pass arguments on the stack we can simply add new arguments to the helper function in the $L L V M-I R$ and replace all the references of the global value representing the stack argument to the newly created function argument. The further steps are independent from the number of arguments:

(1) find the call to the $\mathbf{F}$ _Lifted dummy function

(2) replace the reference of $\mathbf{F} \_$Lifted to the lifted function

(3) inline the call into the helper function

(4) run $L L V M$ 's strongest optimizations.

Based on $L L V M$ 's optimizations we get a clean $L L V M$-IR function that looks vanilla-like as shown in Listing 13. If we compare the input $L L V M-I R$ in Listing 11 and the result in Listing 13, we can see how strong and effective the $L L V M$ optimizations are.

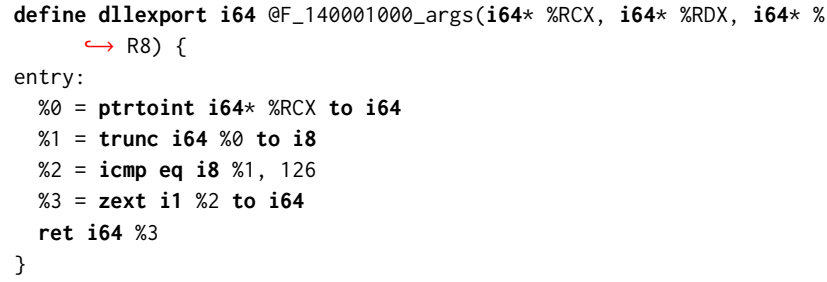

Listing 13: Optimized MBA LLVM-IR function with recovered arguments

\section{EXECUTION}

SATURN is not only able to lift, deobfuscate and brighten the code. It's also able to inject the deobfuscated function back into the input binary. Based on the recovery result (with or without State struct) there are two different ways to call the recovered function.

In both described ways the shared library gets injected into the input binary. In portable executable (PE) files the import table gets replaced with an updated import table that contains an import to a function in our shared library from Section 6.

\subsection{Direct Function Redirection}

When SATURN is able to fully recover the function and its arguments, we can choose to patch the original function and insert a branch instruction to the imported symbol.

\subsection{Context Switch}

If the recovery of the function arguments fails, SATURN is able to keep the State struct in the recovered function. This approach needs a more advanced way to execute the function. The needed runtime is implemented in $\mathbf{C + +}$ and $\mathbf{x 8 6} \mathbf{6 4}$ assembly. The runtime will be compiled into the shared library that is generated in Section 6 . The needed steps to call the function are:

(1) patch an instruction at the beginning of the obfuscated function to push one integer value on the stack (used as function identifier)

(2) patch a second instruction to jump into our imported symbol in the import table.

When the function is reached during execution, it will jump into our runtime that does the following:

(1) create a virtual stack and use it in place of the original one

(2) store all the register values into a local State struct

(3) call the lifted function with the generated State struct

(4) on calls/jumps outside of the lifted function restore the registers from the State struct and handle the return of the function

(5) if the function returns, restore the registers and jump back to the caller

\section{EXPERIMENTAL EVALUATION}

The experiments below seek to answer the following Research Questions:

RQ1 What is the effectiveness on the recovery of the control flow graph?

RQ2 What is the detection rate of the opaque predicates?

RQ3 What is the effectiveness of the deobfuscation?

RQ4 Were all arguments and stack slots recovered?

RQ5 Is the deobfuscated code semantically equivalent to the protected one during execution?

\subsection{Experimental setup}

The attacker has access to SATURN to reverse engineer the given binaries and has the goal to recover the obfuscated functions. For the defense we created some binaries that trigger corner cases and 
Table 3: Results for datasets

\begin{tabular}{|c|c|c|c|c|c|c|c|c|c|c|c|}
\hline \multicolumn{12}{|c|}{ Dataset \#1 } \\
\hline Program & $\begin{array}{l}\text { Time } \\
\text { to lift }\end{array}$ & $\begin{array}{l}\text { Time to } \\
\text { opti- } \\
\text { mize }\end{array}$ & $\begin{array}{c}\text { Detected } \\
\text { Opaque } \\
\text { Predi- } \\
\text { cates }\end{array}$ & $\begin{array}{c}\text { Test } \\
\text { passed }\end{array}$ & $\begin{array}{c}\text { Arguments } \\
\text { recovered } \\
\text { (Lift./Orig.) }\end{array}$ & $\begin{array}{l}\text { Stack } \\
\text { Slots re- } \\
\text { covered }\end{array}$ & $\begin{array}{l}\text { Processed } \\
\text { Instruc- } \\
\text { tions }\end{array}$ & $\begin{array}{c}\text { Recovered } \\
\text { Basic } \\
\text { Blocks }\end{array}$ & $\begin{array}{c}\text { Obfuscation } \\
\text { removed }\end{array}$ & $\begin{array}{l}\text { Solving time } \\
\text { with KLEE } \\
\text { (Lift./Orig.) }\end{array}$ & $\begin{array}{l}\text { Size re- } \\
\text { duction } \\
\text { in Basic } \\
\text { Blocks }\end{array}$ \\
\hline args & $0.541 \mathrm{~s}$ & $0.980 \mathrm{~s}$ & $0 / 0^{a}$ & Yes $^{a}$ & $0 / 6^{a}$ & $0^{a}$ & 110 & $13 / 13$ & Yes & - & 4 \\
\hline cmp_test & $0.408 \mathrm{~s}$ & $0.342 \mathrm{~s}$ & $1 / 1$ & Yes & $2 / 2$ & 7 & 39 & $8 / 8$ & Yes & - & 5 \\
\hline edges & $0.129 \mathrm{~s}$ & $0.233 \mathrm{~s}$ & $2 / 2$ & Yes & $1 / 1$ & 5 & 16 & $4 / 4$ & Yes & - & 3 \\
\hline edges2 & $0.428 \mathrm{~s}$ & $0.622 \mathrm{~s}$ & $0 / 0$ & Yes & $2 / 3^{d}$ & 11 & 120 & $10 / 10$ & Yes & - & 9 \\
\hline $\mathrm{fib}$ & $0.309 \mathrm{~s}$ & $0.287 \mathrm{~s}$ & $0 / 0$ & Yes & $1 / 1$ & 6 & 31 & $4 / 4$ & Yes & - & 0 \\
\hline gotos & $0.599 \mathrm{~s}$ & $0.564 \mathrm{~s}$ & $3 / 3$ & Yes & $3 / 3$ & 10 & 85 & $13 / 13$ & Yes & - & 10 \\
\hline inf_loop & $0.215 \mathrm{~s}$ & $0.285 \mathrm{~s}$ & $1 / 1$ & Yes & $1 / 1$ & 0 & 18 & $2 / 2$ & Yes & - & 0 \\
\hline loop & $0.152 \mathrm{~s}$ & $0.247 \mathrm{~s}$ & $0 / 0$ & Yes & $1 / 1$ & 5 & 21 & $4 / 4$ & Yes & - & 3 \\
\hline multiedges & $0.405 \mathrm{~s}$ & $0.251 \mathrm{~s}$ & $0 / 0$ & Yes & $1 / 1$ & 4 & 21 & $9 / 9$ & Yes & - & 8 \\
\hline op1 & $0.188 \mathrm{~s}$ & $0.010 \mathrm{~s}$ & $1 / 1$ & Yes & $2 / 2$ & 5 & 24 & $3 / 3$ & Partially $^{e}$ & - & 2 \\
\hline tig_virt & $2.337 \mathrm{~s}$ & $1.018 \mathrm{~s}$ & $0 / 0$ & Yes & $1 / 1$ & 17 & 288 & $41 / 41$ & No & - & 23 \\
\hline sse2 & $0.147 \mathrm{~s}$ & $0.429 \mathrm{~s}$ & $0 / 0$ & Yes & $2 / 2$ & 16 & 47 & $1 / 1$ & Yes $^{f}$ & - & 0 \\
\hline \multicolumn{12}{|c|}{ Dataset \#2 } \\
\hline binsec0 & $4.785 \mathrm{~s}$ & $1.144 \mathrm{~s}$ & $0 / 0$ & Yes & $1 / 1$ & 19 & 226 & $25 / 25$ & Yes & $0.43 \mathrm{~s} / 2 \mathrm{~m} 6.30 \mathrm{~s}$ & 9 \\
\hline binsec0_virt & $6.062 \mathrm{~s}$ & $0.595 \mathrm{~s}$ & $0 / 0$ & $\operatorname{Yes}^{a}$ & $0 / 2$ & $0^{a}$ & 464 & $86 / 86$ & No & - & 36 \\
\hline binsec1 & $4.778 \mathrm{~s}$ & $0.141 \mathrm{~s}$ & $0 / 0$ & $\operatorname{Yes}^{a}$ & $0 / 2$ & $0^{a}$ & 273 & $27 / 27$ & Yes & - & 3 \\
\hline forsplit & $3.033 \mathrm{~s}$ & $0.738 \mathrm{~s}$ & $0 / 0$ & Yes & $3 / 3$ & 8 & 112 & $12 / 12$ & Yes & $0.08 \mathrm{~s} / 16.13 \mathrm{~s}$ & 4 \\
\hline \multicolumn{12}{|c|}{ Dataset \#3 } \\
\hline obf0_virt & $27.998 \mathrm{~s}$ & $8.592 \mathrm{~s}$ & $0 / 0$ & $\mathrm{No}^{b}$ & $4 / ?$ & 27 & 2059 & $10 / ?^{c}$ & Yes & - & 4 \\
\hline obf1_func0 & $0.754 \mathrm{~s}$ & $0.680 \mathrm{~s}$ & $11 / 11$ & $\mathrm{No}^{b}$ & $0 / 0$ & 10 & 182 & $12 / 12$ & Yes & - & 10 \\
\hline obf1_func1 & $1.060 \mathrm{~s}$ & $0.827 \mathrm{~s}$ & $0 / 0$ & $\mathrm{No}^{b}$ & $0 / 0$ & 9 & 241 & $14 / 14$ & Yes & - & 13 \\
\hline
\end{tabular}

${ }^{a}$ Remill State struct was used ${ }^{b}$ Binary execution failed because of other protections like anti-tampering

${ }^{c}$ Unknown amount of original basic blocks ${ }^{d}$ Dead argument was detected ${ }^{e}$ MBA formula was not optimized away ${ }^{f}$ Recovered integer arithmetic

use several obfuscation techniques that might hinder binary lifting. Some binaries are protected by Tigress [30], an open source stateof-the-art obfuscator. Some other binaries are protected by real world obfuscators where no source code is available. The machine for the experiments uses Windows 10 Pro x64 on a Intel Core i76700k CPU with 32 GB RAM.

\subsection{Datasets}

We select some small programs that trigger corner cases in several steps of the approach described in this paper. We also choose programs that contain selected obfuscation patterns and real world obfuscated binaries where no source code is available. The datasets and results are available in our online repository ${ }^{2}$.

Dataset \#1. During the development of SATURN we created several test samples that can trigger corner cases. The tests include scenarios like overlapping stack slots, register and stack based arguments, loops, infinite loops, opaque predicates, MBA based opaque predicates and dead code. The programs take an input value from the command line, perform some calculations and print the output to the user. The sample tigress_virtualize is protected with the $t i$ gress virtualization obfuscation pass (-Transform=Virtualize).

Dataset \#2. This dataset includes some programs that were taken from the repository provided by [22] and use the SPLIT and

\footnotetext{
${ }^{2}$ https://github.com/pgarba/Saturn_Results
}

FOR anti-DSE tricks.

Dataset \#3. The last dataset contains two real world binaries that were protected with Obfuscator 0 and Obfuscator $1^{3}$. The binary from Obfuscator1 was chosen because we have an unprotected binary and can easily compare the results. The Obfuscatoro doesn't have an associated unprotected binary, but we choose it because it's a strong obfuscated real world example of a protected virtual machine entry point ${ }^{4}$. Both binaries are heavily obfuscated and were chosen to stress test SATURN.

\subsection{Results \& Observations}

Table 3 shows the results for each tested program in the dataset. In programs for which the argument and stack recovery fails ${ }^{a}$, we are still able to recover the function by staying in the Remill State struct (RQ1). For all other programs the recovery of the arguments and the stack was successful (RQ4). In the programs obfo_func0 and $o b f 1 \_f u n c 0$ we don't know the exact amount of opaque predicates but based on the obfuscator we know that a missed opaque predicate would lead to a broken $L L V M-I R$. For the programs in dataset \#1 and \#2 all opaque predicates are detected (RQ2).

For each program we verified the deobfuscated function for correctness by comparing the output values to the one of the obfuscated program. Programs protected with the Tigress virtualization

\footnotetext{
${ }^{3}$ Obfuscator 0 and Obfuscator 1 are made up names

${ }^{4}$ Context switch from the original $x 86 \_64$ to the virtual context
} 
stay in the virtualized form but the recovered code is clean and readable. The program op1 is only partially deobfuscated. The $M B A$ based opaque predicate is removed but the recovered calculation is still based on the $M B A$ formula ${ }^{e}$. The result of obfo_virt can't be verified but the recovered code is clean, readable and meaningful (RQ3). For the programs in dataset \#2 we are not able to remove the FOR and SPLIT tricks in the Tigress protected samples. In the other programs the tricks are detected and removed.

For dataset \#1 and \#2 we are able to execute the output binary and all the deobfuscated programs behave in a semantically equivalent way to the obfuscated ones (RQ5). For dataset \#3 the recompiled binaries are not working because of some additional anti-tampering checks in those binaries ${ }^{b}$.

For all the programs we verified the output binary obtained by SATURN with the IDA Pro [10] decompiler. The decompiler is returning meaningful and readable pseudo $\mathbf{C}$ code (RQ3). This was failing before as IDA Pro struggles with obfuscated code.

\section{DISCUSSION}

We compared our work to existing $L L V M$ based binary lifting frameworks. All of them were failing in lifting obfuscated code ([36], [15], [20], [14]) as they were not built for this task. A good overview of the existing $L L V M$-based lifters is given in the comparison table that can be found in [20]. One exception is $S 2 E$ [6], the symbolic execution tool based on $Q E M U$ [2]. $S 2 E$ is able to export the generated traces in pure $L L V M$-IR form but, considering it is a dynamic approach, we can't compare it to our work.

We also compared our work to the symbolic execution tool Triton [31]. We were interested to see how SATURN compares to Triton while processing the opaque predicates with an $S M T$ solver. We noticed that $S A T U R N$ is able to create much smaller and optimized $S M T$ queries due to prior optimizations. In this regard our approach is much more efficient compared to the one in Triton and therefore reduces the solving times ${ }^{5}$. This complies with the assumption in [3].

The work presented in [19], although based on binary execution traces, is a valid starting point to improve the detection of the dynamic opaque predicates in SATURN. While the work presented in [3] describes a strong simplification methodology based on the Drill\&foin synthesis technique [1] which is orthogonal to the ones in SATURN and could further improve the MBA expressions handling. As discussed in Section 12, a plugin system would enable us to integrate these approaches during the exploration phase.

\section{RELATED WORK}

Machine Learning. One of the side products of SATURN is a database with normalized opaque predicates and obfuscation patterns thanks to the Redis [24] cache used in the Souper Optimizer [27]. We think that it would be interesting to see what a machine learning based method like the one introduced in the work of Tofighi-Shirazi et al. [32] could make of this information to improve the opaque

\footnotetext{
${ }^{5}$ The comparison is available in the results' repository
}

predicate recovery rate in SATURN.

Exploit generation. Rolles et al. described how $S M T$ solving can help to automatically chain together sequences of ROP gadgets, so that the sequence is semantically equivalent to a model payload [34]. We think that SATURN can be used to create such ROP gadgets and, in combination with a DSE tool like KLEE [4] model the needed sequence and payload for an exploit.

Effectiveness of Synthesis in Concolic Deobfuscation. Biondi et al. summarize in [3] that SMT solvers alone are not efficient enough against $M B A$ based obfuscation. As future work they proposed to study a tool that could drive the concolic execution of obfuscated programs by retrieving a compact representation of obfuscated constraints. They expect that the simplified constraints are easier to solve or check for satisfiability. We believe that SATURN is exactly the tool that they are looking for. It would be interesting to study the work done in SATURN in combination with the work in [3].

\section{CONCLUSION}

In this paper we have proposed a state-of-the-art framework for software deobfuscation based around the LLVM ecosystem. The work implemented in the tool SATURN lifts the attack surface away from the binary level up to the $L L V M-I R$ and solves the problems that appear during binary deobfuscation directly on this level. The results that we reach are not based on any assumptions, instead we use general optimization techniques and $S M T$ solving to extract the control flow graph and deobfuscate the code. The achieved optimized representation can help to apply advanced practical attacks. We believe that the presented work highlights a new perspective on program deobfuscation and complements existing work by lifting the attack surface to a new level.

\section{FUTURE WORK}

We would like to add a plugin system to let the user hook in several phases of the code recovery in SATURN and write their own transformation passes. This could be used to devirtualize a protection like the Tigress virtualization or handle $M B A$ expressions with customized approaches. Right now we are concretizing the stack pointer to be able to retrieve the stack slots, but we think that we could change this step to be based on a completely symbolic approach. We would also like to try out some new ideas in which we change the type of the registers used in Remill into pointers, as this may help to avoid IntToPtr casts in LLVM and generate cleaner code to begin with. Right now we are only able to lift $x 86 \_64$ binary code but Remill also supports lifting of AArch64 and $x 86$ binary code. We are aware that a constant range analysis was recently added to the constant synthesis in Souper [25]. We believe this could be used to tackle the difficulties related with the identification of switch-case destination addresses.

\section{ACKNOWLEDGMENTS}

To Roman, for taking the time to proofread our work and also contributing with his knowledge during countless discussions. To Helge, who cared for guiding me during many years of my career as 
a reverse engineer. To Davide, Joao and Massimo for introducing me to the software protections world and for the endless discussions about reverse engineering.

\section{REFERENCES}

[1] Remis Balaniuk. 2015. Drill and Join: A Method for Exact Inductive Program Synthesis. In Logic-Based Program Synthesis and Transformation, Maurizio Proietti and Hirohisa Seki (Eds.). Springer International Publishing, Cham, 219-237.

[2] Fabrice Bellard. 2005. QEMU, a Fast and Portable Dynamic Translator. In Proceedings of the Annual Conference on USENIX Annual Technical Conference (ATEC '05). USENIX Association, Berkeley, CA, USA, 41-41. http://dl.acm.org/citation. cfm?id=1247360.1247401

[3] Fabrizio Biondi, Sébastien Josse, Axel Legay, and Thomas Sirvent. 2017. Effectiveness of Synthesis in Concolic Deobfuscation. Computers and Security 70 (Sept. 2017), 500-515. https://doi.org/10.1016/j.cose.2017.07.006

[4] Cristian Cadar, Daniel Dunbar, and Dawson Engler. 2008. KLEE: Unassisted and Automatic Generation of High-coverage Tests for Complex Systems Programs. In Proceedings of the 8th USENIX Conference on Operating Systems Design and Implementation (OSDI'08). USENIX Association, Berkeley, CA, USA, 209-224. http://dl.acm.org/citation.cfm?id $=1855741.1855756$

[5] Marek Chalupa. 2016 [cit. 2019-07-12]. Slicing of LLVM Bitcode [online]. Master's thesis. Masaryk University, Faculty of Informatics, Brno. https://theses.cz/id/ ok0jh1/

[6] Vitaly Chipounov, Volodymyr Kuznetsov, and George Candea. 2012. The S2E Platform: Design, Implementation, and Applications. ACM Trans. Comput. Syst 30, 1, Article 2 (Feb. 2012), 49 pages. https://doi.org/10.1145/2110356.2110358

[7] Christian Collberg, Clark Thomborson, and Douglas Low. 1997. A Taxonomy of Obfuscating Transformations. Technical Report 148. Department of Computer Sciences, The University of Auckland. http://www.cs.auckland.ac.nz/ collberg/ Research/Publications/CollbergThomborsonLow97a/index.html

[8] Ron Cytron, Jeanne Ferrante, Barry K. Rosen, Mark N. Wegman, and F. Kenneth Zadeck. 1991. Efficiently Computing Static Single Assignment Form and the Control Dependence Graph. ACM Trans. Program. Lang. Syst. 13, 4 (Oct. 1991), 451-490. https://doi.org/10.1145/115372.115320

[9] Leonardo De Moura and Nikolaj Bjørner. 2008. Z3: An Efficient SMT Solver. In Proceedings of the Theory and Practice of Software, 14th International Conference on Tools and Algorithms for the Construction and Analysis of Systems (TACAS'08/ETAPS'08). Springer-Verlag, Berlin, Heidelberg, 337-340. http://dl. acm.org/citation.cfm?id=1792734.1792766

[10] Chris Eagle. 2008. The IDA Pro Book: The Unofficial Guide to the World's Most Popular Disassembler. No Starch Press, San Francisco, CA, USA.

[11] Pascal Junod, Julien Rinaldini, Johan Wehrli, and Julie Michielin. 2015. ObfuscatorLLVM - Software Protection for the Masses. In Proceedings of the IEEE/ACM 1st International Workshop on Software Protection, SPRO'15, Firenze, Italy, May 19th, 2015, Brecht Wyseur (Ed.). IEEE, 3-9. https://doi.org/10.1109/SPRO.2015.10

[12] Johannes Kinder and Dmitry Kravchenko. 2012. Alternating Control Flow Reconstruction. In Proc. 13th Int. Conf. Verification, Model Checking, and Abstract Interpretation (VMCAI) (LNCS), Vol. 7148. Springer, 267-282.

[13] Johannes Kinder and Helmut Veith. 2008. Jakstab: A Static Analysis Platform for Binaries. In Proc. 20th Int. Conf. Computer Aided Verification (CAV) (LNCS), Vol. 5123. Springer, 423-427.

[14] Lukas Korencik. 2019. Decompiling Binaries into LLVM IR Using McSema and Dyninst. Master's thesis. Masaryk University, Faculty of Informatics, Brno. https: //is.muni.cz/th/pxe1j/

[15] J. Kroustek, P. Matula, and P. Zemek. 2017. RetDec: An Open-Source MachineCode Decompiler. [talk]. Presented at Botconf 2017, Montpellier, FR.

[16] Chris Lattner and Vikram Adve. 2004. LLVM: A Compilation Framework for Lifelong Program Analysis \& Transformation. In Proceedings of the International Symposium on Code Generation and Optimization: Feedback-directed and Runtime Optimization (CGO '04). IEEE Computer Society, Washington, DC, USA, 75http://dl.acm.org/citation.cfm?id=977395.977673

[17] LLVM Mailing List. 2019. Optimization Problem. Retrieved July 12, 2019 from http://llvm.1065342.n5.nabble.com/llvm-dev-Optimization-Problemtd127994.html\#a127998

[18] Microsoft. 2018. x64 calling convention. Retrieved July 13, 2019 from https //docs.microsoft.com/en-us/cpp/build/x64-calling- convention?view=vs- 2019

[19] Jiang Ming, Dongpeng Xu, Li Wang, and Dinghao Wu. 2015. LOOP: Logic Oriented Opaque Predicate Detection in Obfuscated Binary Code. In Proceedings of the $22^{\text {nd }}$ ACM SIGSAC Conference on Computer and Communications Security (CCS '15). ACM, New York, NY, USA, 757-768. https://doi.org/10.1145/2810103. 2813617

[20] Trail of Bits. 2019. McSema - Framework for lifting X86, AMD64, and AARCH64 program binaries to LLVM bitcode. Retrieved August 24, 2019 from https //github.com/trailofbits/mcsema

[21] Trail of Bits. 2019. Remill - Library for lifting of x86, amd64, and aarch64 machine code to LLVM bitcode. Retrieved July 10, 2019 from https://github.com/trailofbits/ remill

[22] Mathilde Ollivier, Sébastien Bardin, Richard Bonichon, and Jean-Yves Marion. 2019. How to Kill Symbolic Deobfuscation for Free; or Unleashing the Potential of Path-Oriented Protections. CoRR (2019). http://arxiv.org/abs/1908.01549

[23] Quarkslab. 2019. Epona - Epona is a new compiler that integrates innovative software protection for code integrity, obfuscation, and tamper-proofing. Retrieved August 27, 2019 from https://epona.quarkslab.com/en/

[24] Redislabs. 2019. Redis: open source, in-memory data structure store, used as a database, cache and message broker. Retrieved August 22, 2019 from https: //redis.io

[25] John Regehr. 2019. Souper github - Use ConstantRange analysis to help Constant Synthesis. Retrieved August 24, 2019 from https://github.com/google/souper/ commit/20b20ef8c8883513a9cc388b5c01743b70033fb5

[26] Thomas Reinbacher and Jörg Brauer. 2011. Precise Control Flow Reconstruction Using Boolean Logic. In Proceedings of the Ninth ACM International Conference on Embedded Software (EMSOFT '11). ACM, New York, NY, USA, 117-126. https: //doi.org/10.1145/2038642.2038662

[27] Raimondas Sasnauskas, Yang Chen, Peter Collingbourne, Jeroen Ketema, Jubi Taneja, and John Regehr. 2017. Souper: A Synthesizing Superoptimizer. CoRR (2017). http://arxiv.org/abs/1711.04422

[28] Sebastian Schrittwieser, Stefan Katzenbeisser, Johannes Kinder, Georg Merzdovnik, and Edgar Weippl. 2016. Protecting Software Through Obfuscation: Can It Keep Pace with Progress in Code Analysis? ACM Comput. Surv. 49, 1, Article 4 (April 2016), 37 pages. https://doi.org/10.1145/2886012

[29] Jiri Slaby. 2016. LLVM Slicer - Static slicer based on the Mark Weiser's algorithm. Retrieved July 12, 2019 from https://github.com/sdasgup3/llvm-slicer

[30] Clark Taylor and Christian Colberg. 2016. A Tool for Teaching Reverse Engineering. In 2016 USENIX Workshop on Advances in Security Education (ASE 16). USENIX Association, Austin, TX. https://www.usenix.org/conference/ase16/workshopprogram/presentation/taylor

[31] Philippe Tillet, H. T. Kung, and David Cox. 2019. Triton: An Intermediate Language and Compiler for Tiled Neural Network Computations. In Proceedings of the 3rd ACM SIGPLAN International Workshop on Machine Learning and Programming Languages (MAPL 2019). ACM, New York, NY, USA, 10-19. https://doi.org/10.1145/3315508.3329973

[32] Ramtine Tofighi-Shirazi, Irina Măriuca Asăvoae, Philippe Elbaz-Vincent, and Thanh-Ha Le. 2019. Defeating Opaque Predicates Statically through Machine Learning and Binary Analysis. In 3rd International Workshop on Software PROtection. London, United Kingdom. https://hal.archives-ouvertes.fr/hal-02269192

[33] Linda Torczon and Keith Cooper. 2011. Engineering A Compiler (2nd ed.). Morgan Kaufmann Publishers Inc., San Francisco, CA, USA.

[34] Julien Vanegue, Sean Heelan, and Rolf Rolles. 2012. SMT Solvers for Software Security. In Proceedings of the 6th USENIX Conference on Offensive Technologies (WOOT'12). USENIX Association, Berkeley, CA, USA, 9-9. http://dl.acm.org/ citation.cfm?id=2372399.2372412

[35] Shuai Wang, Pei Wang, and Dinghao Wu. 2015. Reassembleable Disassembling. In Proceedings of the 24th USENIX Conference on Security Symposium (SEC'15). USENIX Association, Berkeley, CA, USA, 627-642. http://dl.acm.org/citation. cfm?id=2831143.2831183

[36] S. Bharadwaj Yadavalli and Aaron Smith. 2019. Raising Binaries to LLVM IR with MCTOLL (WIP Paper). In Proceedings of the 20th ACM SIGPLAN/SIGBED International Conference on Languages, Compilers, and Tools for Embedded Systems (LCTES 2019). ACM, New York, NY, USA, 213-218. https://doi.org/10.1145/ 3316482.3326354

[37] B. Yadegari, B. Johannesmeyer, B. Whitely, and S. Debray. 2015. A Generic Approach to Automatic Deobfuscation of Executable Code. In 2015 IEEE Symposium on Security and Privacy. 674-691. https://doi.org/10.1109/SP.2015.47

[38] Yingzhou Zhang. 2019. SymPas: Symbolic Program Slicing. CoRR (2019). http: //arxiv.org/abs/1903.05333 